EMS Annual Meeting Abstracts

Vol. 18, EMS2021-11, 2021, updated on 20 Sep 2021

https://doi.org/10.5194/ems2021-11

EMS Annual Meeting 2021

(C) Author(s) 2021. This work is distributed under

the Creative Commons Attribution 4.0 License.

\title{
The role of knowledge networks in facilitating the creation of climate information services
}

\author{
Francesca Larosa ${ }^{1,2}$ and Marta Bruno-Soares ${ }^{3}$ \\ ${ }^{1}$ Ca' Foscari University, Economics, Economics, Italy \\ ${ }^{2}$ Euro-Mediterranean Center on Climate Change \\ ${ }^{3}$ University of Leeds
}

Knowledge networks are collections of individuals and teams who work together across organizational, spatial and disciplinary boundaries to invent and share a body of knowledge. Climate services are tools and application that support decision-making by transforming raw climate data into tailored information. They call for co-development practices in place and for successful collaboration between different stakeholders. Knowledge networks for climate services are intermediaries that facilitate the interaction between upstream (providers) and downstream (user) actors operating at various scales (local, national, regional and supranational). They assist the decision-making process of a wide set of users by creating windows of opportunity and by delivering usable climate information. The aim of this work is to frame and assess the efficiency of knowledge networks for climate services in promoting innovation and facilitate its diffusion. First, we characterize knowledge networks learning from insights of a multidisciplinary literature. Second, we analyse the purpose, the process and the audience of each knowledge network for climate services by screening their programmatic documents. We then assess the efficiency of knowledge networks by performing content analysis of interviews with knowledge network managers and by checking for the existence of inconsistencies or gaps with the initial objectives. We find knowledge networks for climate services pursue four objectives: coordination, innovation promotion, science-policy interface and support to members. We also find inadequate tools to monitor the members activities, but a strong positioning within the climate services domain. On the communication side, knowledge networks for climate services mostly interact with developers of climate services but they face challenges in sharing the members' activities with users. Our work fills a significant knowledge gap and helps providing new tools of performance assessment in absence of a clearly defined methodology. The identification of bottlenecks and under-performing mechanisms in the climate information services sphere allows the elaboration of strategies to improve the status quo and facilitates the diffusion of these innovations. 